\title{
Finite-bias electronic transport of molecules in a water solution
}

\author{
I. Rungger, ${ }^{1}$ X. Chen, ${ }^{1}$ U. Schwingenschlögl, ${ }^{2}$ and S. Sanvito ${ }^{1}$ \\ ${ }^{1}$ School of Physics and CRANN, Trinity College, Dublin 2, Ireland \\ ${ }^{2}$ PSE Division, KAUST, Thuwal 23955-6900, Kingdom of Saudi Arabia
}

(Received 25 January 2010; published 4 June 2010)

\begin{abstract}
The effects of water wetting conditions on the transport properties of molecular nanojunctions are investigated theoretically by using a combination of empirical-potential molecular-dynamics and first-principles electronic-transport calculations. These are at the level of the nonequilibrium Green's-function method implemented for self-interaction corrected density-functional theory. We find that water effectively produces electrostatic gating to the molecular junction with a gating potential determined by the time-averaged water dipole field. Such a field is large for the polar benzene-dithiol molecule, resulting in a transmission spectrum shifted by about $0.6 \mathrm{eV}$ with respect to that of the dry junction. The situation is drastically different for carbon nanotubes (CNTs). In fact, because of their hydrophobic nature the gating is almost negligible so that the average transmission spectrum of wet $\mathrm{Au} / \mathrm{CNT} / \mathrm{Au}$ junctions is essentially the same as that in dry conditions. This suggests that CNTs can be used as molecular interconnects also in water-wet situations, for instance, as tips for scanning tunnel microscopy in solution or in biological sensors.
\end{abstract}

DOI: $10.1103 /$ PhysRevB.81.235407

PACS number(s): 73.63.Fg, 73.20.At

\section{INTRODUCTION}

Electron transfer in biological systems is rather different from electron transport in an electronic device. In addition to the intrinsic materials differences between the respective conducting media, namely, soft molecules in biology against inorganic semiconductors in electronics, the electron transport in these two situations differs for the effects and the relevance of the environment. In fact, while one wants to keep electronic devices in dry conditions to avoid electrostatic disorder, biological electron transfer is characterized by a time-evolving local dielectric environment.

The boundary between the two fields, electronics and biology, becomes however blurred when one looks at the nanoscale. On the one hand, "conventional" nanotechnology has expanded in the biology domain with a growing number of electronic applications requiring operation in wet conditions. In addition to scanning tunnel microscopy in water, ${ }^{1}$ which has been available for the last two decades, several biological sensors have been recently proposed. These, for instance, include cancer markers detectors ${ }^{2}$ and protocols for DNA sequencing. ${ }^{3}$ On the other hand, when a solution is confined at the nanoscale, highly ordered structures can form at room temperature. ${ }^{4}$ This essentially means that under such stringent confinement a biological system almost behaves like a solid.

Interestingly, numerous experiments to date on electronic transport through molecules are carried out in solution. ${ }^{5,6}$ Still, with a few exceptions, ${ }^{7-9}$ most of the theoretical calculations are performed in the dry, i.e., without explicitly including water molecules in the simulations. Therefore the question on how water affects the current-voltage $(I-V)$ characteristic in a molecular junction remains. In general one may expect that solutions made with polar molecules, such as $\mathrm{H}_{2} \mathrm{O}$, may affect significantly the transport because of the generation of local dipole fields. However, since the time scale of a transport measurement is far longer than the typical molecular rearrangement of a solution, one should ask what is the time-averaged dipolar field near the molecule of interest. This, together with the degree of localization of the relevant molecular orbitals, will determine whether or not the wetting conditions influence the junction electronic transport.

In order to address those fundamental questions we have performed a number of combined empirical-potential molecular-dynamics (MD) and quantum transport simulations for molecular junctions in water. Our computational strategy is to investigate first the dynamics of $\mathrm{H}_{2} \mathrm{O}$ and then to evaluate the electronic transport of a representative number of configurations, i.e., for a number of MD snapshots. In particular, we have considered two rather different junctions, both using gold electrodes but differing for the local charge arrangement of the molecule of interest. The first is made from a polar molecule, namely, benzene-dithiol (BDT), while the second includes locally charge neutral carbon nanotubes (CNTs), in particular a $(3,3)$ metallic and a $(8,0)$ insulating one.

We find that the effect of water on the transport is that of effectively gating the molecule, therefore shifting almost rigidly its transmission spectrum. This is rather pronounced for BDT, but only tiny in the case of CNTs, and reflects the different charge distribution of the two classes of molecules. We associate those differences to the time-averaged dipole field of the water. Notably all the calculations are performed with density-functional theory (DFT) including appropriately corrections for the electronic structure of water. This is an essential condition for quantitative predictions.

The paper is organized as follows. In the next section we describe the device setup for the calculations and briefly the computational tools used. Then we analyze the transport. First we look at the electronic structure of a gold capacitor with water wetting the two electrodes and then we consider finite-bias conductance across BDT and the CNTs. Finally we present our main conclusions.

\section{METHODS}

Empirical-potential MD calculations are performed with the NAMD2 package ${ }^{10}$ parametrized by following the standard 
additive CHARMM force-field protocol. ${ }^{11}$ BDT is attached to the two gold electrodes at the $\mathrm{Au}(111)$ hollow site, which has been previously calculated to be the low-energy bonding position for BDT on $\mathrm{Au}(111) .{ }^{12}$ We define our coordinate system with the $z$ axis along the (111) direction and the $x-y$ plane orthogonal to it. All the calculations are carried out with periodic boundary conditions in the $x-y$ plane. For water we adopt TIP3P model ${ }^{13}$ as implemented in CHARMM, which includes Lennard-Jones parameters for the hydrogen atoms as well as the oxygen atom. ${ }^{14}$ This yields excellent structural parameters for the water molecule, namely, a $\mathrm{O}-\mathrm{H}$ bond length of $0.9572 \AA$ a H-O-H bond angle of $104.52^{\circ}$, a bond force constant of $K_{b}=450 \mathrm{kcal} \mathrm{mol}^{-1} \AA^{-2}$ and an angle force constant of $K_{\theta}=55 \mathrm{kcal} \mathrm{mol}^{-1} \mathrm{rad}^{-2}$.

By using the VMD package ${ }^{15}$ solvation procedure, 83 water molecules are initially added to a $(25 \times 17.3 \times 7.3) \AA^{3}$ box intercalated between the $\mathrm{Au}$ electrodes. Note that the dimension along the $z$ axis is chosen in such a way that the van der Waals distance between the $\mathrm{Au}(111)$ surface and the water molecules is taken into account. After equilibration the extension of the water box along the $z$ direction shrinks to an average length of $5.7 \AA$. This gives a final water density of approximately $1.07 \mathrm{~g} / \mathrm{cm}^{3}$. Since our main objective is that of examining the effects of water over the conductance of an $\mathrm{Au} / \mathrm{molecule/Au}$ device, we always fix the atomic positions of both the molecule and the electrodes. The interaction between the $\mathrm{H}_{2} \mathrm{O}$ molecules and gold is treated at the level of a 12-6 Lennard-Jones potential,

$$
U^{\mathrm{LJ}}=4 \epsilon\left[\left(\frac{\sigma}{r}\right)^{12}-\left(\frac{\sigma}{r}\right)^{6}\right]
$$

with parameters for $\mathrm{Au} \quad(\epsilon=0.039 \mathrm{kcal} / \mathrm{mol}$ and $\sigma$ $=2.934 \AA)$ taken from the literature ${ }^{16}$ Periodic boundary conditions are applied with a cutoff of $12 \AA$ for long-range interactions. In order to maintain the size and shape of the cell constant, we perform simulations in the microcanonical ensemble with reinitialized velocities to $300 \mathrm{~K}$ for every 1000 time steps and with a time step of $2 \mathrm{fs}$. The trajectory is recorded every 4 ps from the initial equilibration of $1 \mathrm{~ns}$ to a total simulation time of $20 \mathrm{~ns}$. For the systems comprising the CNTs, $\mathrm{Au}(111) / \mathrm{CNT}(3,3)-\mathrm{H}_{2} \mathrm{O} / \mathrm{Au}(111) \quad$ and $\mathrm{Au}(111) / \mathrm{CNT}(8,0)-\mathrm{H}_{2} \mathrm{O} / \mathrm{Au}(111)$, and for the reference $\mathrm{Au}(111) / \mathrm{H}_{2} \mathrm{O} / \mathrm{Au}(111)$ capacitor, similar conditions and procedures are followed. The parameters for the aromatic carbon atoms are used for CNTs.

For each system we calculate the transport properties for a set of representative MD configurations taken after equilibration. From these we can then estimate the fluctuations in the transmission and current over time as well as their timeaveraged values. We use the SMEAGOL $a b$ initio electronictransport code to calculate the zero-bias transmission coefficients and the $I-V$ characteristics. SMEAGOL combines the nonequilibrium Green's-function method with DFT (Refs. 17-19) and has the pseudopotential code SIESTA (Ref. 20) as its electronic-structure platform. Note that here in the MD simulations we do not include contributions to the forces acting on the water molecules arising from the external electric field. However, a separate calculation done with an external electric field of $1 \mathrm{~V} / \mathrm{nm}$ returned an average water distribution rather similar to that obtained when no electric field is applied so that the effect on the transport is expected to be weak. This problem will be investigated in details in the future.

The local-density approximation (LDA) to the exchange and correlation functional is adopted throughout. The atomic self-interaction correction (ASIC) (Ref. 21) scheme, however, is used for the water and the BDT molecule, in order to bring their ionization potentials (IPs) in closer agreement to experiments. This has been already proved to be a successful strategy for aligning correctly the highest occupied molecular orbital (HOMO) energy, $\epsilon_{\mathrm{HOMO}}$, of the molecule under consideration to the Fermi level $\left(E_{\mathrm{F}}\right)$ of the electrodes. ${ }^{12,22}$ Note that the same corrections also reproduce well the band gap of many insulating oxides, after the ASIC potential is rescaled appropriately. ${ }^{21}$ Such a rescaling in bulk crystals is attributed to charge screening, which in solids is usually stronger than in molecules. In general we use a scaling parameter, $\alpha$, ranging between 0 and 1 , to adjust the amount of self-interaction correction included ( $\alpha=0$ corresponds to the LDA, $\alpha=1$ is the full ASIC). Usually $\alpha$ is 1 for small molecules, it is around 0.5 for insulating oxides, and it vanishes for metals. For this reason ASIC is never applied to Au.

For the transport calculations, we use a real-space mesh cutoff of $200 \mathrm{Ry}$ and an electronic temperature of $300 \mathrm{~K}$. The unit cell includes five $\mathrm{Au}$ atomic layers on each side of the $\mathrm{Au}(111)$ surface, which are enough to screen charging at the Au-molecule interface. In order to reduce the system size the Au $5 d$ shell is kept in the core so that we consider only $6 s$ orbitals in the valence. In Ref. 12 , it is shown that the use of such an approximation gives results that are in good agreement with those obtained including the $5 d$ Au orbitals in the valence. We use a single- $\zeta$ basis for Au $6 s$, specifically optimized to give the correct work function for the $\mathrm{Au}(111)$ surface. $^{23}$ The rest of the basis set is double $\zeta$ for the $\mathrm{C} s$ and $\mathrm{C} p$ orbitals, and double $\zeta$ plus polarization for $\mathrm{S}(s$ and $p)$. For the $\mathrm{H}_{2} \mathrm{O}$ molecules we use a double- $\zeta$ basis for both $\mathrm{H}$ and O. However, when calculating transport through CNTs we reduce it to single $\zeta$ in order to keep the size of the density matrix tractable.

The charge density is obtained by splitting the integral of the Green's function into a contribution calculated over the complex energy plane and one along the real axis. ${ }^{17,18}$ The complex part of the integral is computed by using 16 energy points on the complex semicircle, 16 points along the line parallel to the real axis and 16 poles. The integral over real energies necessary at finite bias is evaluated over at least 1000 points $^{17,18}$ per electron volt.

\section{AU CAPACITOR IN WATER}

In a transport calculation it is crucial to describe accurately both the electrodes' work function, $W$, and the IP of the molecule, so that the correct alignment of the molecular levels to the electrodes' $E_{\mathrm{F}}$ is reproduced. With this in mind we first analyze the electronic structure of water sandwiched between Au electrodes. Such a setup corresponds to calculating the electronic structure of an Au parallel plate capacitor, where the two plates are separated by water. The unit cell, 




FIG. 1. (Color online) Au capacitor with water as dielectric medium. The top panel shows the unit cell used for the MD simulations, which includes $\mathrm{H}_{2} \mathrm{O}$ confined between the two $\mathrm{Au}$ electrodes. In panel (a), we show the probability to find a $\mathrm{O}$ (solid curve) or a $\mathrm{H}$ (dashed curve) atom at a given $z$ position. Panels (b)-(d) are the planar averages of the Hartree electrostatic potential $\bar{V}_{H}$ as function of position along the transport direction, $z$, for different setups: (b) entire junction, (c) junction without the electrodes, and (d) junction without water. The gray curves, merging in a shadow, are the results for 21 snapshots taken at different times after equilibration, the black solid curves are their time averages. The dashed curve in (c) shows the difference between the time averages of panels (b) and (d).

containing $408 \mathrm{H}_{2} \mathrm{O}$ molecules and $480 \mathrm{Au}$ atoms, is shown in Fig. 1. The statistical distribution of $\mathrm{H}_{2} \mathrm{O}$ about the $\mathrm{Au}$ plates is described in Fig. 1(a), where we plot the normalized probability, $p(z)$, to find $\mathrm{O}$ (solid curve) or $\mathrm{H}$ (dashed curve) at a given position $z$ in the cell $\left[\int p(z) d z=1\right]$. Such a distribution is obtained by using the MD data for all the time steps included in the $16 \mathrm{~ns}$ simulation after equilibration. We note that $p(z)$ is constant in the middle of the gap between the plates, indicating an average random arrangement of the $\mathrm{H}_{2} \mathrm{O}$ molecules. In contrast close to the $\mathrm{Au}$ interface there are marked oscillations in $p(z)$, signaling a correlation of the water position with respect to the Au surface. Note that the peaks in $p(z)$ are found at the same positions for $\mathrm{O}$ and $\mathrm{H}$ atoms. This indicates that on average there is no net dipole at the interface.

Next the Au work function is calculated by using the same Au parallel plate capacitor of Fig. 1 after we have removed the water, i.e., with vacuum as spacer between the plates. $W$ is then the energy difference between $E_{\mathrm{F}}$ and the vacuum potential, $V_{\text {vacuum. }}$. Such an exercise is reported in Fig. 1(d) where we show the planar average $\bar{V}_{H}$ of the electrostatic Hartree potential along the $x-y$ plane. Note that the absolute value of $\bar{V}_{H}$ in Au is arbitrary since it depends on the pseudopotentials. However, in the middle of the capacitor $\bar{V}_{H}$ $=V_{\text {vacuum }}$. Therefore, setting $V_{\text {vacuum }}=0$ we have $W=-E_{\mathrm{F}}$. We find $W=5.3 \mathrm{eV}$, in good agreement with experiments.

We now turn our attention to the electronic structure of water. This must be extracted for its liquid phase, i.e., from

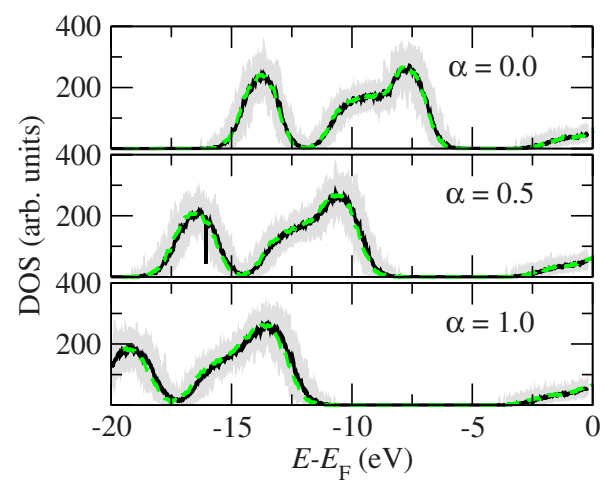

FIG. 2. (Color online) DOS projected onto $\mathrm{H}_{2} \mathrm{O}$ for the capacitor of Fig. 1 and obtained for different values of the ASIC scaling parameter $\alpha$. The gray lines are the superimposed curves for $21 \mathrm{MD}$ snapshots and the solid black one is their average. The dashed curve is the average DOS for the same MD snapshots for a system where $\mathrm{Au}$ is replaced by vacuum.

the MD simulations. We consider data averaged over 21 structural configurations corresponding to 21 equally spaced MD snapshots. The so-calculated planar average of the electrostatic potential is shown in Fig. 1(b). The gray lines, merging in a shadow, are the superimposed curves for all the snapshots while the black line is their time average. We note oscillations of $\bar{V}_{H}$ close to Au. These are due to the arrangement of the water molecules with respect to the Au surface. In contrast in the middle of the junction $\bar{V}_{H}$ is rather flat due to the average random orientation of the molecules [see also Fig. 1(a)].

From the same simulations we can extract the IP of liquid water. In transport one wants $\epsilon_{\mathrm{HOMO}}$ of the molecule of interest to correspond to the actual negative of its IP. ${ }^{24,25} \mathrm{Al}-$ though this should be the case for exact DFT, it happens only rather rarely in practice for the standard local approximations of the exchange and correlation functional. ${ }^{26}$ In the case of liquid $\mathrm{H}_{2} \mathrm{O}$ the experimental value of the IP ranges between 9.3 (Ref. 27) and $9.9 \mathrm{eV}^{28}$ This is in rather good agreement with recent $a b$ initio calculations of the electron removal energy. ${ }^{29,30}$

We evaluate $\epsilon_{\mathrm{HOMO}}$ for liquid $\mathrm{H}_{2} \mathrm{O}$ by calculating the density of states (DOS) projected onto the water molecules. This is shown in Fig. $2\left(V_{\text {vacuum }}=0\right)$, where again the gray curves are the superimposed data for all the MD snapshots and the black one is their average. We note that there are large fluctuations in the DOS over time with energy shifts on the order of $1 \mathrm{eV}$. The average value, however, is smooth and can be reliably taken as the liquid water DOS. In this way the top of the water "valence band" is calculated to be only $-6 \mathrm{eV}$ in LDA, in agreement with previous DFT calculations, ${ }^{31}$ but still far from the experimental value.

We then apply ASIC to $\mathrm{H}_{2} \mathrm{O}$ and find that $\epsilon_{\mathrm{HOMO}}$ moves to $-8.5 \mathrm{eV}$ for $\alpha=0.5$ and to $-11 \mathrm{eV}$ for $\alpha=1.0$ so that $\alpha$ $=0.7$ fits the average experimental value $(-9.5 \mathrm{eV})$. Such an optimal value, as usually with ASIC, ${ }^{21}$ in general improves the entire electronic structure and returns a gap between the highest occupied water orbital and the lowest unoccupied water orbital of about $6.4 \mathrm{eV}$, in good agreement with the experimental value of $6.9 \mathrm{eV}^{32}$ Note that $\alpha=0.7$ is typical for moderately ionic insulating oxides. ${ }^{21}$ 


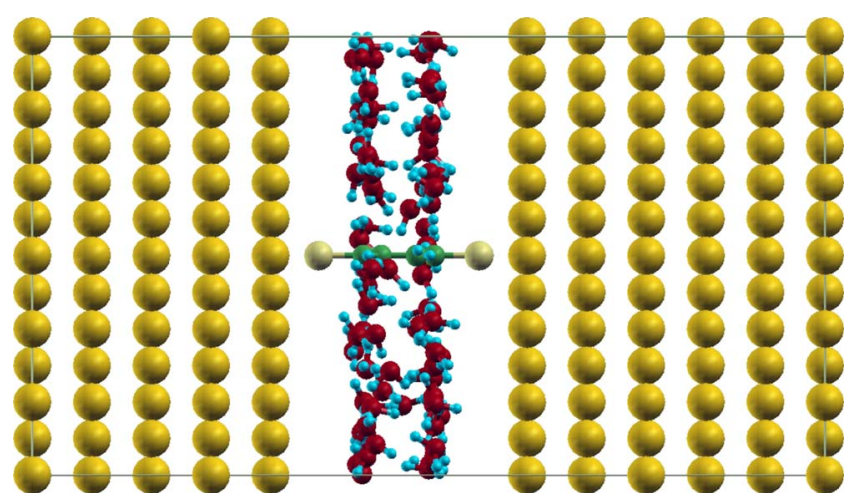

FIG. 3. (Color online) Unit cell used for the BDT molecule in water attached to $\mathrm{Au}$ electrodes.

In order to analyze the effects of the $\mathrm{Au} / \mathrm{H}_{2} \mathrm{O}$ interface over the water IP and DOS we perform a second set of calculations, where we remove the Au plates and we replace them by vacuum. This corresponds to a hypothetical $\mathrm{H}_{2} \mathrm{O}$ slab. In this case [see Fig. 1(c)] $\bar{V}_{H}$ at a given MD time step has a finite slope in the vacuum region, which is caused by the noncompensated dipoles at the $\mathrm{H}_{2} \mathrm{O}$ external surface. These dipoles produce a long-range electric field outside slab, so that $V_{\text {vacuum }}$ of a single snapshot is not defined. However, the time-averaged $\bar{V}_{H}$ (black curve) is approximately flat in the vacuum, demonstrating that, although at each time step surface charge may lead to long-ranged electric fields, its time average is actually zero.

If we now take the average $\bar{V}_{H}$ away from the water molecules as $V_{\text {vacuum }}$, we can plot the time-averaged DOS for the water slab and superimpose it to that calculated for the $\mathrm{Au} / \mathrm{H}_{2} \mathrm{O} / \mathrm{Au}$ capacitor (see Fig. 2). We find that the two DOSs overlap on each other, confirming our results for the water IP and the fact that on average there is little electronic interaction between $\mathrm{Au}$ and $\mathrm{H}_{2} \mathrm{O}$. Our results also suggest that one should ideally use periodic boundary conditions to simulate the electronic structure of molecules in solution. These eliminate the possible spurious electric fields in the vacuum, which can lead to an unphysical rearrangement of the energy levels. Furthermore, for simulations of $\mathrm{H}_{2} \mathrm{O}$ surfaces it is essential to consider time averages so that the water electric field vanishes in vacuum. Figure 1(c) also shows the difference between the time-averaged $\bar{V}_{H}$ of the $\mathrm{Au}$ capacitors with and without water (dashed line). This difference is almost identical to the time-averaged $\bar{V}_{H}$ for the water slab and is consistent with the fact that the $\mathrm{Au}$ electrodes do not induce any noticeable change in the average DOS of $\mathrm{H}_{2} \mathrm{O}$.

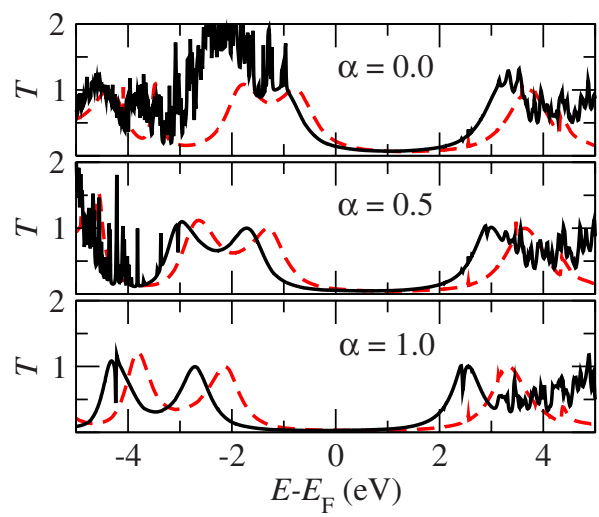

FIG. 4. (Color online) Transmission coefficient for transport through BDT in water as function of energy for one MD snapshot and for different values of the ASIC scaling parameter $\alpha$. The dashed curves are for BDT in dry conditions (no water) while the solid curves are for BDT in solution.

\section{BENZENE-DITHIOL}

Electron transport through BDT attached to Au electrodes has been extensively studied both experimentally $y^{5,33-35}$ and theoretically. ${ }^{12,22}$ In fact, because of its simple structure, BDT is an ideal system for comparing theory with experiments. However, also for BDT the LDA description is not adequate and it is necessary to employ ASIC. ${ }^{12,22}$ In order to limit the number of adjustable parameters we set the same $\alpha$ for both BDT and $\mathrm{H}_{2} \mathrm{O}$ and check that such a value reproduces well previous transport calculations for the $\mathrm{Au} /$ BDT/Au junction in dry conditions. ${ }^{12,22}$

We first investigate the $V=0$ transport (the cell used is shown in Fig. 3). In Fig. 4, the transmission coefficient $T(E ; V=0)$ for one MD snapshot is shown as function of energy, $E$, for different values of the ASIC scaling parameter $\alpha$ (solid curves). The same quantity is compared to that calculated for the same cell, this time without including water (dashed curves). In Table I the values of $T\left(E_{\mathrm{F}}\right)$ are given for the different parameters. In general, the effects of water are twofold: first there is a shift of the BDT transmission peaks to lower energies, and second there appear additional sharp transmission peaks, which are attributed to resonant transport through the electronic states of $\mathrm{H}_{2} \mathrm{O}$. Interestingly the height, the width, and the relative position of the transport peaks with respect to each other is unchanged when water is present.

Therefore, the main effect of adding water is to shift the energy levels of BDT so that water acts as an external gate. Since the BDT molecular orbitals extend over the entire mol-

TABLE I. Transmission coefficient at the Fermi energy $\left[T\left(E_{\mathrm{F}}\right)\right]$ for different values of the ASIC scaling parameter $\alpha$. The transmission values at $E_{\mathrm{F}}$ for $\alpha=0.0,0.5$, and 1.0 are for the same MD snapshot used in Fig. 4 , whereas for $\alpha=0.7$ the value given corresponds to the time-averaged transmission of Fig. 7 .

\begin{tabular}{lccccc}
\hline \hline$\alpha$ & 0.0 & 0.5 & 1.0 & 0.7 & Expt. \\
\hline$T\left(E_{\mathrm{F}}\right)\left(\right.$ no $\left.\mathrm{H}_{2} \mathrm{O}\right)$ & 0.22 & 0.078 & 0.031 & 0.053 & \\
$T\left(E_{\mathrm{F}}\right)\left(\right.$ with $\left.\mathrm{H}_{2} \mathrm{O}\right)$ & 0.14 & 0.056 & 0.030 & 0.040 & $0.011^{\mathrm{a}}$ \\
\hline \hline
\end{tabular}

${ }^{\mathrm{a} R e f e r e n c e} 5$. 


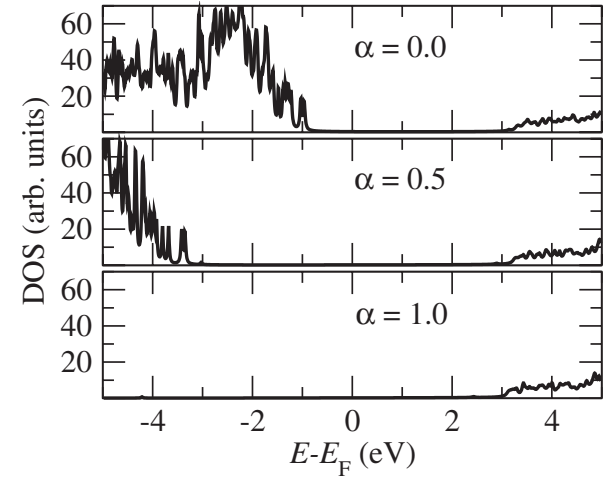

FIG. 5. Density of states projected onto $\mathrm{H}_{2} \mathrm{O}$ for the junction of Fig. 3 as function of energy for one MD snapshot and for different values of the ASIC scaling parameter $\alpha$.

ecule and are strongly coupled to $\mathrm{Au},{ }^{12}$ all the levels shift by approximately the same amount. If the levels were more localized, we might have expected a change in their relative position, sensitively dependent on the local configuration of $\mathrm{H}_{2} \mathrm{O} .{ }^{36}$

We now analyze in more detail the electronic properties of $\mathrm{H}_{2} \mathrm{O}$ for the same MD snapshot. In Fig. 5 the DOS projected onto the water molecules is shown over the same energy range as that of $T$ in Fig. 4. It is clear that the additional peaks in the transmission (Fig. 4) are at energies where $\mathrm{H}_{2} \mathrm{O}$ has a finite DOS, confirming that these are due to transport through the electronic states of water. The additional transmission peaks below $E_{\mathrm{F}}$ are rather close to the Fermi level when $\alpha=0$, whereas they move down in energy as $\alpha$ is increased. In contrast the position of the peaks above $E_{\mathrm{F}}$ is almost constant for different $\alpha$ since the ASIC mainly affects occupied states. We also find that for $\alpha=0$ the water HOMO is about $-6.3 \mathrm{eV}$ from $V_{\text {vacuum }}$ while it is at $-8.7 \mathrm{eV}$ for $\alpha$ $=0.5$. These values agree with the values obtained for the water slab.

The self-consistent current-voltage, $I-V$, curve is shown next in Fig. 6 for the same MD snapshot and for different values of $\alpha$. Generally speaking the presence of water leads to a reduction in the current, which is more pronounced for

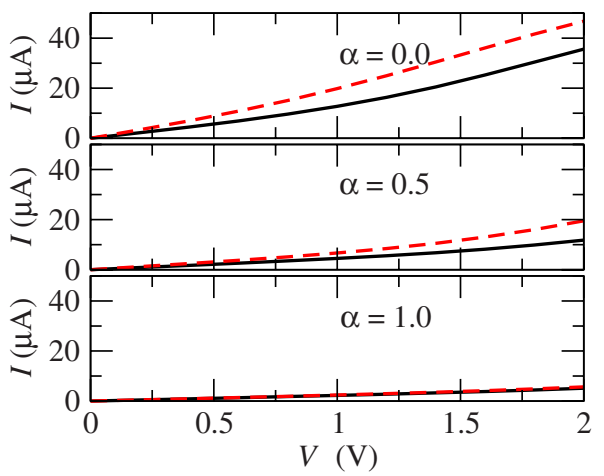

FIG. 6. (Color online) Current, $I$, as function of the bias voltage, $V$, through the BDT molecule calculated at the first MD time step and for different values of the SIC scaling parameter $\alpha$. The dashed curves are for the molecule in the dry while the solid curves are for BDT in solution. small $\alpha$. The reason for such a reduction is that $\epsilon_{\mathrm{HOMO}}$ is very close to $E_{\mathrm{F}}$ for small $\alpha$ so that a tiny shift of the BDT levels to lower energies considerably reduces $T\left(E_{\mathrm{F}} ; V \approx 0\right)$. For $\alpha=1$ there is almost no change in the current, since $E_{\mathrm{F}}$ is approximately at midgap already in dry conditions.

We now briefly discuss the most appropriate value of $\alpha$ in this context. For BDT in the gas phase $\alpha=1$ gives $\epsilon_{\mathrm{HOMO}}$ close to the experimental IP. ${ }^{21,22}$ However, when BDT is immersed in water additional screening lowers down the value of $\alpha$. We then take $\alpha=0.7$ (the optimal value for $\mathrm{H}_{2} \mathrm{O}$ ), which provides a good IP for water and also accounts for the additional screening in BDT due to the solution. Importantly our results depend little on the exact choice of $\alpha$, as long as it is on the order of 0.5 , i.e., such that $\epsilon_{\mathrm{HOMO}}$ for $\mathrm{H}_{2} \mathrm{O}$ is well below the $\mathrm{Au} E_{\mathrm{F}}$. Based on the zero-bias DOS one might expect that in an LDA calculation there would be an enhanced conductance for voltages above $1 \mathrm{~V}$.

We now move to calculate the time-averaged transmission coefficient and the $I-V$ curves. In this case $T(E ; V=0)$ and the $\mathrm{H}_{2} \mathrm{O}$ DOS are evaluated over 201 snapshots taken in the last 16 ns of our MD simulations while the $I-V$ curves are evaluated over only 21 . Transmission and DOS are presented in Fig. 7, where again the curves for the single snapshot calculations are plotted in gray to form a shadow, while their average is a solid black line. In general, when $\mathrm{H}_{2} \mathrm{O}$ is introduced in the simulation, there is a rigid shift of the entire spectrum toward lower energies with respect to the dry situation (dashed line). This is because BDT and $\mathrm{H}_{2} \mathrm{O}$ are both polar molecules and in time the water molecules arrange around BDT so to screen the local dipole field. Such screening moves the average BDT molecular levels to lower energies.

This analysis is confirmed in Fig. 8(a), where we present the $\mathrm{O}$ and $\mathrm{H}$ position distributions along the $y$ direction, for those $\mathrm{H}$ and $\mathrm{O}$ atoms lying either above or below the plane of the BDT (shadowed region in Fig. 8). Note that we define the $y$ axis as the direction perpendicular to the plane of the BDT. In contrast to the case of the Au capacitor, now the $p(y)$ 's of $\mathrm{O}$ and $\mathrm{H}$ ions differ near BDT. In particular, we find that $\mathrm{H}$ approaches BDT approximately $1 \AA$ closer than O. This means that on average the first solvation layer is oriented with the $\mathrm{H}$ atoms of $\mathrm{H}_{2} \mathrm{O}$ molecules pointing toward the BDT, as suggested by elementary electrostatics, since the

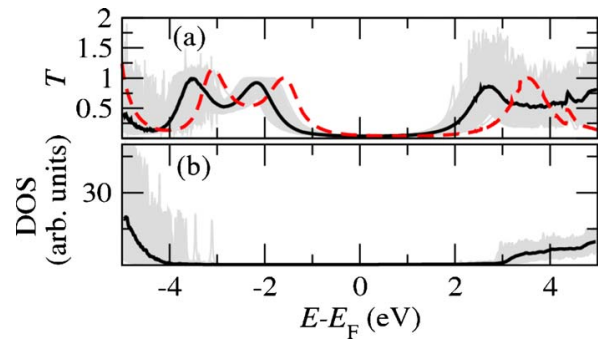

FIG. 7. (Color online) Time averaged (a) transmission coefficient and (b) DOS projected onto the $\mathrm{H}_{2} \mathrm{O}$ molecules for BDT attached to Au. The calculations are obtained with ASIC and $\alpha$ $=0.7$; the solid black curves are the time averages over 201 time steps while the gray ones forming a shadow are for each of the 201 time steps. The dashed curve is for the dry situation. 


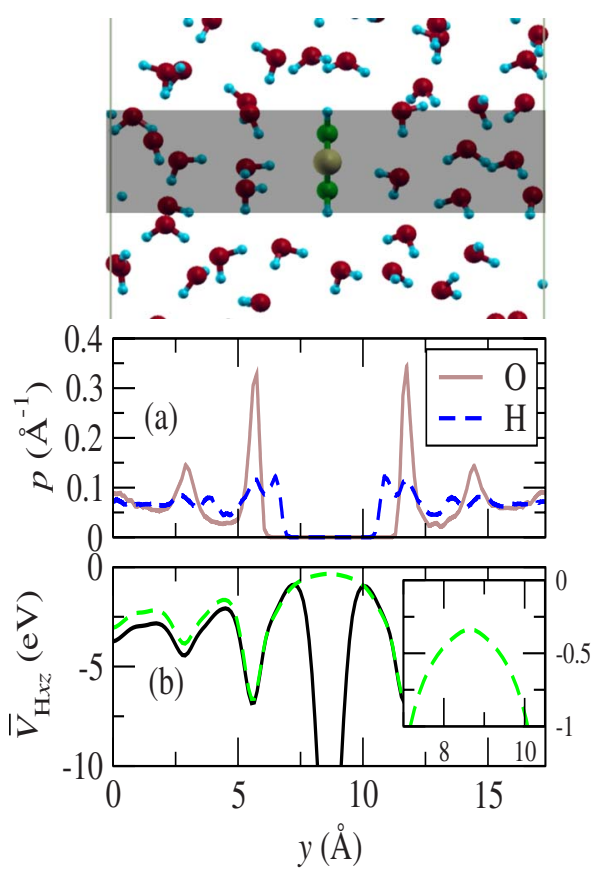

FIG. 8. (Color online) (a) Probability to find an O (solid curve) or an $\mathrm{H}$ (dashed curve) atom at a given position $y$ in the $\mathrm{Au} /$ $\mathrm{BDT} / \mathrm{Au}$ junction, for atoms whose $x$ coordinate lies within the shadowed region. In the top panel we show a representative snapshot of the atomic configuration of the water. Note that in the first solvation layer the $\mathrm{H}_{2} \mathrm{O}$ molecules are oriented with the $\mathrm{H}$ atoms pointing toward the BDT. (b) The black curve shows the time average of the planar average over $x$ and $z$ of the electrostatic potential, $\bar{V}_{H x z}$, which is averaged for $x$ laying in the shadowed region, and for the $z$ coordinate extending between the two $\mathrm{S}$ atoms of the BDT. The dashed green curve illustrates the difference of this planar average between the system with and the one without water. The inset is a zoom in of this difference in the region around the BDT where no water molecules are found.

$\mathrm{C}$ and $\mathrm{S}$ atoms are fractionally negatively charged, while the $\mathrm{H}$ atoms have a positive charge. The second peak of the $\mathrm{H}$ atoms overlaps with the first peak of $\mathrm{O}$, indicating that while one of the $\mathrm{H}$ atoms points toward the BDT, the second one aligns with the negative $\mathrm{O}$ atoms. It is also interesting to note that the $\mathrm{O}$ distribution has a second pronounced peak in addition to that close to the BDT, signaling a relative large degree of order also in the second solvation layer.

The effect of such a dipole on the electrostatic potential can be seen in Fig. 8(b). Here we plot the time average of the electrostatic potential averaged over $x$ and $z$, where $x$ lies in the shadowed region in the figure and $z$ lies between the two $\mathrm{S}$ atoms of the BDT. The black curve shows this potential average, whereas the dashed green curve shows the difference between the potential average with water and the one without water. In the region where the BDT molecule lies, and which does not contain water molecules (for $y$ between about 7 and $10 \AA$ ), this difference is negative, with a value around $-0.5 \mathrm{eV}$. This corresponds approximately to the shift of the spectrum to lower energies due to the water.

We finally turn our attention to the fluctuations. Generally, time fluctuations in the position of the BDT single-particle levels result in zero-bias conductance fluctuations. ${ }^{7}$ These

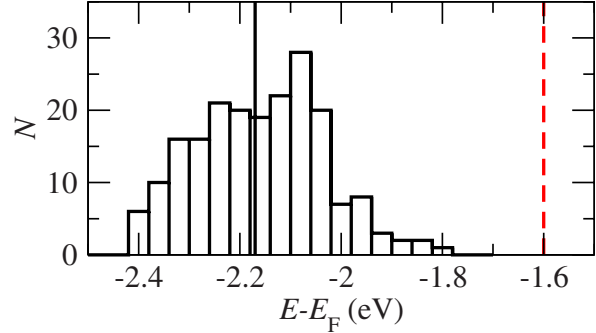

FIG. 9. (Color online) Histogram of $\epsilon_{\mathrm{HOMO}}$ extracted from the maximum in $T(E)$ at around $-2 \mathrm{eV}$ (see Fig. 7) for $\alpha=0.7$ and for $201 \mathrm{MD}$ time steps. $N$ is the number of times $\epsilon_{\mathrm{HOMO}}$ is found in the given energy window specified by the bin width. The solid line indicates the time-averaged $\epsilon_{\mathrm{HOMO}}$ while the dashed red one marks the result in dry conditions.

cause both a reduction in the average height of the various transmission peaks and at the same time an increase in their widths. For BDT attached to Au this second effect is rather small, since the transmission peaks at each time step are already rather broad, due to the strong electronic coupling with the electrodes. However, we expect that for molecules weakly coupled to the electrodes and thus presenting sharp peaks in $T(E)$ this effect will be more pronounced, probably dominating the energy-level broadening.

As already mentioned before the electrostatic interactions of water with the BDT mimics a gate potential. At each MD time step such an effective gate voltage changes depending on the relative position of $\mathrm{H}_{2} \mathrm{O}$. In order to quantify the fluctuations of the BDT molecular levels we track the position of $\epsilon_{\mathrm{HOMO}}[$ from the peak in $T(E)]$ over time, and display the result in the form of a histogram in Fig. 9. In the plot $N$ is the number of counts $\epsilon_{\mathrm{HOMO}}$ is found in a particular energy window, the dashed red line indicates the position of $\epsilon_{\mathrm{HOMO}}$ in the dry while the solid black line indicates the timeaveraged $\epsilon_{\mathrm{HOMO}}$ in $\mathrm{H}_{2} \mathrm{O}$ solutions. Clearly $\epsilon_{\mathrm{HOMO}}$ fluctuates between -1.8 and $-2.4 \mathrm{eV}$, i.e., in an energy range of 0.6 $\mathrm{eV}$. The time-averaged $\epsilon_{\mathrm{HOMO}}$ is about $0.6 \mathrm{eV}$ below $\epsilon_{\mathrm{HOMO}}$ for the dry molecule, which means that the effective waterinduced gating potential is about $0.6 \mathrm{eV}$. We note that these results are obtained for room-temperature MD. At lower temperatures, we expect a reduction in the conductance fluctuations although the average shift in the transmission spectrum is likely to remain rather similar.

Finally, in Fig. 10 we present the self-consistent $I-V$ characteristics, where we conclude that the current fluctuates about its time average by approximately $\pm 20 \%$ while it is reduced from that in the dry by about $35 \%$. This discussion is based on ASIC calculations with the optimal value of $\alpha$ $=0.7$. As discussed previously, the most appropriate correction for BDT in the dry is $\alpha \approx 1$. If the same correction is exported into the wet situation the reduction in the current due to water becomes almost negligible.

\section{CARBON NANOTUBES}

We now move to the analysis of the effects of $\mathrm{H}_{2} \mathrm{O}$ wetting on nonpolar molecules, i.e., molecules presenting local charge neutrality. In particular, we choose two different 




FIG. 10. (Color online) $I-V$ curve for a BDT molecule attached to gold in presence of water obtained with ASIC $(\alpha=0.7)$; the solid black curve corresponds to the average current over 21 time steps, the gray curves merging in a shadow are the $I-V$ curves of each individual configuration and the red dashed curve is for BDT in dry conditions.

CNTs: (i) $(3,3)$ metallic armchair and (ii) $(8,0)$ insulating zigzag.

In the case of the metallic $(3,3)$ CNT we again perform 20 ns long MD simulations and calculate the observables over 201 equally spaced snapshots in the last $16 \mathrm{~ns}$. The unit cell used is shown in Fig. 11 for one particular MD snapshot. This has a $(20.0 \times 17.3) \AA^{2}$ cross section and contains 480 $\mathrm{Au}$ atoms, $192 \mathrm{C}$ atoms, and $360 \mathrm{H}_{2} \mathrm{O}$ molecules. The AuCNT distance is simply obtained by adding the $\mathrm{Au}$ and $\mathrm{C}$ atomic radii (respectively, 1.44 and $0.7 \AA$ ) and it is close to that obtained by total-energy minimization. ${ }^{37}$ We note that the exact conformation of the Au-CNT bonding is not known and that changes in bond structure lead to quantitative changes in the transmission spectra. ${ }^{38}$ Here however we are mainly interested in investigating how the transmission is affected by the water so that the precise bonding geometry is less important.

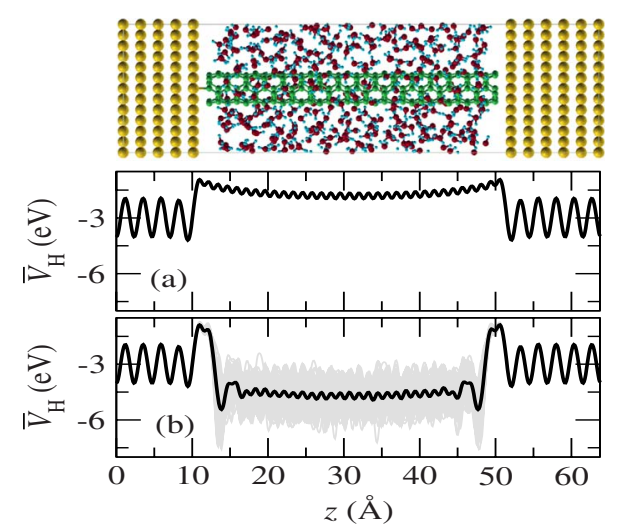

FIG. 11. (Color online) Au/CNT/Au junction. The top panel shows the unit cell used for the MD simulations, which includes the CNT, Au electrodes, and water molecules. In panels (a) and (b) we present the planar averages of the Hartree electrostatic potential, $\bar{V}_{H}$, as function of position along the transport direction $z$ : (a) junction without water and (b) junction with water. The gray curves, merging in a shadow, are the results for all 201 MD snapshots, the black solid curves are their time averages.

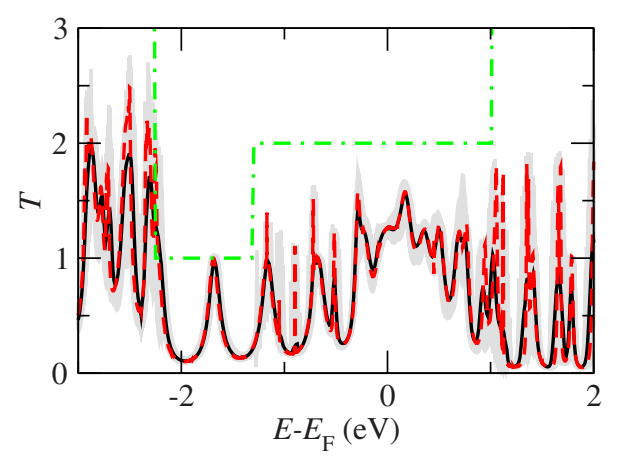

FIG. 12. (Color online) Transmission coefficient as function of energy for the $\mathrm{Au}(111) / \mathrm{CNT}(3,3)-\mathrm{H}_{2} \mathrm{O} / \mathrm{Au}(111)$ junction. The solid black curve corresponds to the average $T$ over 201 MD snapshots, the dashed red curve is the transmission for the same junction in the dry and the dot-dashed green line indicates the number of channels (spin degenerate) for the infinite CNT. The gray curves, merging in a shadow, are $T(E ; V=0)$ for each of the MD snapshot.

As already mentioned before because of the large system size here we use a single- $\zeta$ basis for $\mathrm{H}_{2} \mathrm{O}$. We verified that this gives a similar IP to that obtained with the double- $\zeta$ basis. In what follows we will use $\alpha=0.7$ for $\mathrm{H}_{2} \mathrm{O}$, but no ASIC for the CNTs, since their electron screening is good. We have verified that the band structure of the $(3,3)$ CNT agrees well with previous calculations. ${ }^{39}$ In particular, we obtain a CNT work function of $4.4 \mathrm{eV}$ in good agreement with previous calculations. ${ }^{40}$ The IP for $(3,3)$ CNT is not available experimentally, but that of similar CNTs ranges between 4.8 and $5.0 \mathrm{eV}^{41-43}$ thus is not far from what calculated here.

Since $W$ of Au is about $1 \mathrm{eV}$ larger than that of the CNT, electrons transfer from the CNT into Au, leading to a substantial band bending. This is demonstrated in Fig. 11(a), where the planar average of the Hartree potential is plotted for the $\mathrm{Au} / \mathrm{CNT} / \mathrm{Au}$ junction in dry conditions. Close to the $\mathrm{Au} / \mathrm{CNT}$ interface the oscillating $\bar{V}_{H}$ is higher than in the middle of the junction, whereas for an infinite CNT $\bar{V}_{H}$ oscillates around a constant average. We note that such charging effects have been neglected in previous tight-binding calculations. ${ }^{38}$ However charging leads to a shift in the transmission spectrum so that it is important to include such an effect in a self-consistent way.

Next we look at the wet situation of Fig. 11(b). In this case $\bar{V}_{H}$ for a single MD snapshot oscillates dramatically along the CNT. However, when the time average is considered [solid black curve in Fig. 11(b)] a regular pattern emerges, where $\bar{V}_{H}$ resembles closely that obtained in the dry. This confirms that on average the position of the $\mathrm{H}_{2} \mathrm{O}$ molecules away from the interface is random. Since CNTs are hydrophobic, we expect the interaction between the water and the CNT to be weak. This is confirmed by taking the difference between $\bar{V}_{H}$ calculated with and without $\mathrm{H}_{2} \mathrm{O}$ molecule and observing that the resulting curve matches closely that of the water slab calculated previously [see Fig. 1(c)].

The transmission coefficients for all the 201 MD snapshots are shown in Fig. 12 as superimposed gray curves together with their average (solid black line), the same quantity 


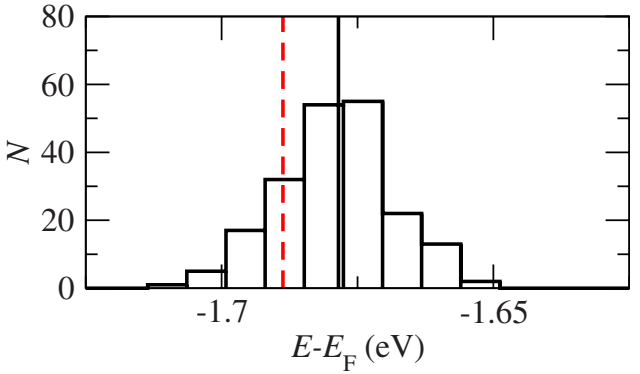

FIG. 13. (Color online) Histogram of the energy position of the transmission peak located at $-1.7 \mathrm{eV}$ below $E_{\mathrm{F}}$ (see Fig. 12). The histogram has been constructed from 201 MD snapshots. $N$ is the number of times the peak is found in the given energy window specified by the bin width. The solid line indicates the timeaveraged position while the dashed red one marks the result in dry conditions.

calculated in dry conditions (dashed red line), and the total number of open channel in the CNT (dot-dashed green line). In the figure we shift $E_{\mathrm{F}}$ in such a way that $\bar{V}_{H}$ for the infinite CNT matches $\bar{V}_{H}$ of the CNT attached to Au without water in the middle of the junction. The necessary shift is about 0.8 $\mathrm{eV}$, which correctly corresponds to the difference in the work functions between the CNT and Au.

The main result is that the average transmission in wet conditions and that of the dry junction overlap almost exactly, demonstrating that in this case $\mathrm{H}_{2} \mathrm{O}$ has no gating effect. This can be easily understood by recalling that, since the CNT has no polar edges, the average $\mathrm{H}_{2} \mathrm{O}$ conformation presents no net electrical dipole, so that on average there is no shift of the CNT energy levels. Of course, each individual MD snapshot displays a dipole and the CNT energy levels get shifted. This leads to fluctuations in the transmission. As a result of the dipole fluctuations, we find that some of the sharp transmission peaks visible in the dry are broadened up and have an average reduced height in solution. In some extreme cases (see, for instance, the sharp peak at about $-1 \mathrm{eV}$ ) they are completely washed out by the fluctuations.

Again, in order to quantify the fluctuations of $T(E)$, we choose a particular molecular level (transmission peak) and follow its time fluctuations. Here we select the well-defined peak at $-1.7 \mathrm{eV}$ below $E_{\mathrm{F}}$ and present its energy distribution histogram in Fig. 13. This time the peak position fluctuates over the tiny energy range of $0.06 \mathrm{eV}$, which is one order of magnitude smaller than that of the HOMO of BDT (see Fig. 9). The origin of such small fluctuations is twofold: first the interaction between $\mathrm{H}_{2} \mathrm{O}$ and the CNT is very weak due to the hydrophobic nature of the nanotube and second the CNT $\pi$-like molecular states are delocalized so that local fluctuations in the electrostatic potential largely cancel out over the entire molecule. We also find that the difference between the average peak position (solid black line in Fig. 13) and that in the dry (dashed red line) is only $0.01 \mathrm{eV}$. This is also much smaller then the same quantity calculated for BDT $(0.6 \mathrm{eV})$.

Finally we discuss results for the insulating $(8,0)$ CNT. The simulation cell is identical to that of the $(3,3)$ case but this time we have $288 \mathrm{C}$ atoms and $322 \mathrm{H}_{2} \mathrm{O}$ molecules. The

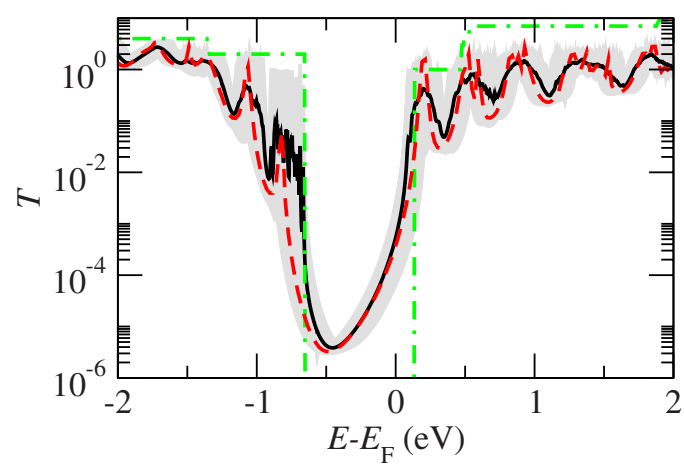

FIG. 14. (Color online) Transmission coefficient as function of energy for the $\mathrm{Au}(111) / \mathrm{CNT}(8,0)-\mathrm{H}_{2} \mathrm{O} / \mathrm{Au}(111)$ junction. The solid (black) curve corresponds to the average $T$ over 41 MD snapshots, the dashed (red) curve is the transmission for the same junction in the dry, and the dot-dashed (green) line indicates the number of channels (spin degenerate) for the infinite CNT. The gray curves, merging in a shadow, are $T(E ; V=0)$ for each of the MD snapshot.

MD simulations are run for $20 \mathrm{~ns}$ and only 41 snapshots are taken within the last $16 \mathrm{~ns}$. We have reduced the number of snapshots from 201 to 41 because this time we do not perform a detailed statistical analysis of the peak position. Our main results are shown in Fig. 14, where we present $T(E ; V=0)$ for all the snapshots (gray lines), their average (solid black line), that in the dry conditions (dashed red line) together with the total number of scattering channels. The transmission coefficient is plotted in logarithmic scale in order to emphasize the tunneling behavior in the gap. In general, the quantitative features of the $\mathrm{Au} / \mathrm{CNT}(8,0)-\mathrm{H}_{2} \mathrm{O} / \mathrm{Au}$ junction are similar to those of the $\mathrm{Au} / \mathrm{CNT}(3,3)-\mathrm{H}_{2} \mathrm{O} / \mathrm{Au}$ one. In particular, also here the average transmission almost overlaps with that of the dry junction meaning that there is a negligible average gating.

However, for energies corresponding to the CNT gap, the transmission fluctuations are rather large due to the tunneling nature of the transport. For instance, $T\left(E_{\mathrm{F}} ; V=0\right)$ fluctuates between $6.0 \times 10^{-5}$ and $2.0 \times 10^{-3}$ within the $41 \mathrm{MD}$ snapshots considered. This means that in tunneling conditions the presence of $\mathrm{H}_{2} \mathrm{O}$ produces substantial variations in the instantaneous conductance amplitude. However and most importantly the time-averaged transmission at $E_{\mathrm{F}}$ is only about $30 \%$ larger than that of the dry junction. This gives us the important result that in general the transport in CNTs is little affected by $\mathrm{H}_{2} \mathrm{O}$ solution regardless of the metallic state of the CNT.

\section{CONCLUSIONS}

In conclusion, we have investigated the effects of water on the transport properties of two types of molecules. This is done by combining empirical-potential molecular dynamics with $a b$ initio electron-transport calculations. First, as an important technical result, we find that self-interaction corrections are fundamental for describing the $\mathrm{H}_{2} \mathrm{O}$ ionization energy and its band gap. This is a prerequisite for quantitative transport calculations. Then our main result is the finding that the $\mathrm{H}_{2} \mathrm{O}$-wetting conditions effectively produce electro- 
static gating to the molecular junction with a gating potential determined by the time-averaged water dipole field. Such a field is rather large for the polar BDT molecule, resulting in an average transmission spectrum shifted by about $0.6 \mathrm{eV}$ with respect to that of the dry junction. In contrast, the hydrophobic nature of the CNTs leads to almost negligible gating, so that the average transmission spectrum for $\mathrm{Au} /$ $\mathrm{CNT} / \mathrm{Au}$ is essentially the same as that in dry conditions, regardless of the CNT metallic state. This suggests that CNTs can be used as molecular interconnects also in water wet situations, for instance, as tips for scanning tunnel microscopy in solutions or in biological sensors.

\section{ACKNOWLEDGMENTS}

This work is sponsored by Science Foundation of Ireland (Grants No. 07/RFP/PHYF235 and No. 07/IN.1/1945) and by the EU FP7 (NANODNA). Computational resources have been provided by KAUST. We thank C. D. Pemmaraju for useful discussions.
${ }^{1}$ S.-L. Yau, C. M. Vitus, and B. C. Schardt, J. Am. Chem. Soc. 112, 3677 (1990).

${ }^{2}$ M. Ferrari, Nat. Rev. Cancer 5, 161 (2005).

${ }^{3}$ P. K. Gupta, Trends Biotechnol. 26, 602 (2008).

${ }^{4}$ K. B. Jinesh and J. W. M. Frenken, Phys. Rev. Lett. 101, 036101 (2008).

${ }^{5}$ X. Xiao, B. Xu, and N. J. Tao, Nano Lett. 4, 267 (2004).

${ }^{6}$ L. Venkataraman, J. E. Klare, C. Nuckolls, M. S. Hybertsen, and M. L. Steigerwald, Nature (London) 442, 904 (2006).

${ }^{7}$ A. Tawara, T. Tada, and S. Watanabe, Phys. Rev. B 80, 073409 (2009).

${ }^{8}$ E. Leary, H. Hobenreich, S. J. Higgins, H. van Zalinge, W. Haiss, R. J. Nichols, C. M. Finch, I. Grace, C. J. Lambert, R. McGrath, and J. Smerdon, Phys. Rev. Lett. 102, 086801 (2009).

${ }^{9}$ H. Cao, J. Jiang, and Y. Luo, J. Am. Chem. Soc. 130, 6674 (2008).

${ }^{10}$ J. C. Phillips, R. Braun, W. Wang, J. Gumbart, E. Tajkhorshid, E. Villa, C. Chipot, R. D. Skeel, L. Kale, and K. Schulten, J. Comput. Chem. 26, 1781 (2005).

${ }^{11}$ A. D. MacKerell, Jr., B. R. Brooks, C. L. Brooks III, L. Nilsson, B. Roux, Y. Won, and M. Karplus, in The Encyclopedia of Computational Chemistry, edited by P. von Ragué Schleyer, P. R. Schreiner, N. L. Alinger, T. Clark, J. Gasteiger, P. Kollmann, and H. F. Schaefer III (Wiley, Chichester, UK, 1998), p. 271.

${ }^{12}$ C. Toher and S. Sanvito, Phys. Rev. B 77, 155402 (2008).

${ }^{13}$ W. L. Jorgensen, J. Chandrasekhar, J. D. Madura, R. W. Impey, and M. L. Klein, J. Chem. Phys. 79, 926 (1983).

${ }^{14}$ W. E. Reiher III, Ph.D. thesis, Harvard University, 1985.

${ }^{15}$ W. Humphrey, A. Dalke, and K. Schulten, J. Mol. Graphics 14, 33 (1996).

${ }^{16}$ Q. Pu, Y. Leng, X. Zhao, and P. T. Cummings, Nanotechnology 18, 424007 (2007)

${ }^{17}$ A. R. Rocha, V. M. Garcia-Suarez, S. W. Bailey, C. J. Lambert, J. Ferrer, and S. Sanvito, Nature Mater. 4, 335 (2005).

${ }^{18}$ A. R. Rocha, V. M. Garcia-Suarez, S. Bailey, C. Lambert, J. Ferrer, and S. Sanvito, Phys. Rev. B 73, 085414 (2006).

${ }^{19}$ I. Rungger and S. Sanvito, Phys. Rev. B 78, 035407 (2008).

${ }^{20}$ J. M. Soler, E. Artacho, J. D. Gale, A. Garcia, J. Junquera, P. Ordejon, and D. Sanchez-Portal, J. Phys.: Condens. Matter 14, 2745 (2002).

${ }^{21}$ C. D. Pemmaraju, T. Archer, D. Sanchez-Portal, and S. Sanvito,
Phys. Rev. B 75, 045101 (2007).

${ }^{22}$ C. Toher and S. Sanvito, Phys. Rev. Lett. 99, 056801 (2007).

${ }^{23}$ C. D. Pemmaraju, I. Rungger, and S. Sanvito, Phys. Rev. B 80, 104422 (2009).

${ }^{24}$ C. Toher, A. Filippetti, S. Sanvito, and K. Burke, Phys. Rev. Lett. 95, 146402 (2005).

${ }^{25}$ C. Toher, I. Rungger, and S. Sanvito, Phys. Rev. B 79, 205427 (2009).

${ }^{26}$ J. F. Janak, Phys. Rev. B 18, 7165 (1978); J. P. Perdew, R. G. Parr, M. Levy, and J. L. Balduz, Jr., Phys. Rev. Lett. 49, 1691 (1982); J. P. Perdew and M. Levy, ibid. 51, 1884 (1983).

${ }^{27}$ T. Watanabe and H. Gerischer, J. Electroanal. Chem. 122, 73 (1981).

${ }^{28}$ B. Winter and M. Faubel, Chem. Rev. 106, 1176 (2006).

${ }^{29}$ C. Millot and B. J. Costa Cabral, Chem. Phys. Lett. 460, 466 (2008).

${ }^{30}$ P. Cabral do Couto and B. J. Costa Cabrala, J. Chem. Phys. 126, 014509 (2007).

${ }^{31}$ D. Prendergast, J. C. Grossman, and G. Galli, J. Chem. Phys. 123, 014501 (2005).

${ }^{32}$ J. V. Coe, A. D. Earhart, M. C. Cohen, G. J. Hoffman, H. W. Sarkas, and K. H. Bowen, J. Chem. Phys. 107, 6023 (1997).

${ }^{33}$ M. A. Reed, C. Zhou, C. J. Muller, T. P. Burgin, and J. M. Tour, Science 278, 252 (1997).

${ }^{34}$ M. Tsutsui, Y. Teramae, S. Kurokawa, and A. Sakai, Appl. Phys. Lett. 89, 163111 (2006).

${ }^{35}$ T. Dadosh, Y. Gordin, R. Krahne, I. Khivrich, D. Mahalu, V. Frydman, J. Sperling, A. Yacoby, and I. Bar-Joseph, Nature (London) 436, 677 (2005).

${ }^{36}$ I. T. Suydam and S. G. Boxer, Biochemistry 42, 12050 (2003).

${ }^{37}$ J. J. Palacios, A. J. Perez-Jimenez, E. Louis, E. SanFabian, and J. A. Verges, Phys. Rev. Lett. 90, 106801 (2003).

${ }^{38}$ I. Deretzis and A. La Magna, Nanotechnology 17, 5063 (2006).

${ }^{39}$ K. P. Bohnen, R. Heid, and C. T. Chan, J. Phys.: Condens. Matter 21, 084206 (2009).

${ }^{40}$ W. S. Su, T. C. Leung, and C. T. Chan, Phys. Rev. B 76, 235413 (2007).

${ }^{41}$ M. Shiraishi and M. Ata, Carbon 39, 1913 (2001).

${ }^{42}$ R. Gao, Z. Pan, and Z. L. Wang, Appl. Phys. Lett. 78, 1757 (2001).

${ }^{43}$ S. Suzuki, C. Bower, Y. Watanabe, and O. Zhou, Appl. Phys. Lett. 76, 4007 (2000). 